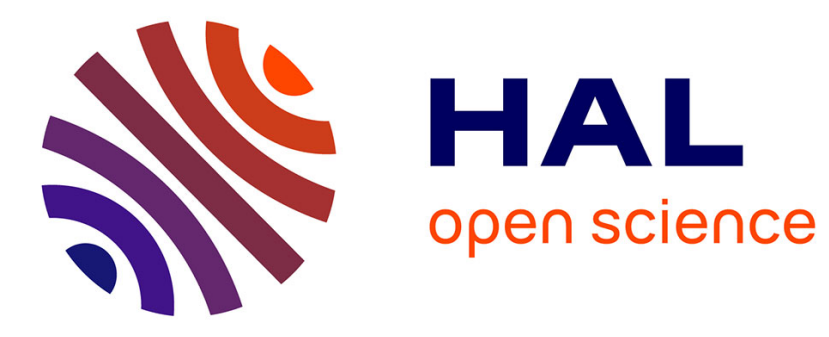

\title{
L'initiation bouddhique au mariage. Formes rituelles en Birmanie
}

François Robinne

\section{To cite this version:}

François Robinne. L'initiation bouddhique au mariage. Formes rituelles en Birmanie. Aséanie, Sciences humaines en Asie du Sud-Est, 2002, 10 (10), pp.11-37. halshs-01866897

\section{HAL Id: halshs-01866897 https://shs.hal.science/halshs-01866897}

Submitted on 3 Sep 2018

HAL is a multi-disciplinary open access archive for the deposit and dissemination of scientific research documents, whether they are published or not. The documents may come from teaching and research institutions in France or abroad, or from public or private research centers.
L'archive ouverte pluridisciplinaire HAL, est destinée au dépôt et à la diffusion de documents scientifiques de niveau recherche, publiés ou non, émanant des établissements d'enseignement et de recherche français ou étrangers, des laboratoires publics ou privés. 


\title{
L'initiation bouddhique au mariage
}

\section{Formes rituelles en Birmanie}

\author{
François Robinne
}

\section{L'aptitude spirituelle au mariage}

Ni le faire-part d'une cérémonie bouddhique de prise de robe - à l'issue $\checkmark$ de laquelle les jeunes adolescents restent quelques jours au monastèreet le faire-part de mariage sont, en Birmanie, construits sur le même modèle, c'est que les relations hommes femmes sont essentielles dans la structuration de ces deux cérémonies. De fait, un certain nombre de similitudes les rapprochent. Spiro (1971, 240 et 1986, 185) fait par exemple remarquer que des "rituels du seuil "” associés au paiement d'une "rançon" interviennent dans le déroulement de l'une et de l'autre cérémonie:

The custom of interrupting a ceremonial event until a ransom is paid is not confined, it should be noted, to the wedding. It is also found, for example, in the Buddhist ordination rite in which the procession of the young novice is obstructed until a ransom is paid.

Dans leurs formes rituelles tout du moins (les seules envisagées ici), les cérémonies bouddhiques de prise de robe et laïque de mariage présentent une sorte de complémentarité. Mais la corrélation entre les deux rituels n'est pas seulement factuelle. J'ai eu l'occasion de montrer que, au-delà de l'initiation bouddhique qu'est d'abord la prise de robe, la seule dimension

1. D'après l'expression employée par A. Van Gennep (1981). 
reconnue par mes informateurs, cette cérémonie consacre l'aptitude spirituelle au mariage (Robinne 2000, 201-233). Je voudrais cette fois aller plus avant dans la démonstration et considérer la cérémonie initiatique de prise de robe en regard des matériaux ethnographiques réunis ces dernières années sur la cérémonie du mariage ${ }^{2}$. Du point de vue développé ici, les deux cérémonies non seulement font état de concordances inhérentes à la sphère d'influence bouddhique à laquelle elles participent, mais elles s'éclairent aussi l'une l'autre dans leur composante laïque. C'est donc dans leur mise en perspective que sont envisagés dans ce qui suit la prise de robe et le mariage.

La cérémonie de prise de robe qu'est censé faire tout bouddhiste à son adolescence est appelée shinbyu en birman (rhaí pru dans sa forme translitérée). Traduite par "devenir seigneur", l'expression recouvre une valeur sémantique très large. En fin de phrase, le titre shin est une forme de politesse qu'adressent les femmes à leur interlocuteur masculin ou féminin ${ }^{3}$. Il est également appliqué, en début de phrase, à l'intention des esprits (nat), des rois birmans, des sawbwa ou princes shan, du Bouddha (avant et après l'Éveil) et de ses disciples; c'est par ce titre encore que sont désignés, lors d'une cérémonie de prise de robe, les jeunes princes en puissance (rhaí loñ:, prononcé "shinlaung", mot à mot "seigneur + futur") avant de devenir novices (kuirañ, prononcé "koyin", sāmaṇe dans la forme dérivée du pāli). Judson $(1966,866)$ illustre l'usage de ce mot en prenant pour exemple l'expression pāli-birmane rhañ moń sāmaṇe (mot à mot: vénérable + petit frère + novice). Dans tous les cas, shin est un terme honorifique, quelle que soit la nature roturière, princière ou religieuse de la personne ainsi désignée.

C'est de l'un à l'autre de ces statuts auxquels sont successivement élevés les enfants lors de la cérémonie du shinbyu. Pendant toute la durée du rituel, les enfants sont habillés, portés et vénérés comme des princes à qui l'on vient rendre hommage. Une "servante" (thin:, prononcé "thein") leur est

2. Depuis 1994, j'ai eu l'occasion d'assister à un grand nombre de prises de robe; des enquêtes systématiques ont été faites en 1994 au village de Myinkaba à Pagan, en 1995 et en 1998 à Inlé, en 2001 à Mandalay. Les enquêtes sur les mariages ont été effectuées principalement à Mandalay en 2000.

3. Sous cette forme, rhaí correspond à la translittération de la graphie birmane actuelle. Mais les travaux du professeur Than Tun $(1978,94-95)$ montrent que le mot apparaît dans les inscriptions de Pagan sous la forme syan. Le passage de "sy" à "rh" semble être commun. Il s'observe, par exemple, dans les formes écrites de l'ethnonyme Shan qui s'écrivait autrefois syam: avant de devenir l'actuel rham: ou rhan:. Le même auteur fait remarquer que le terme kuirañ pour novice n'était pas utilisé entre le $\mathrm{X}^{\mathrm{e}}$ et le XIII ${ }^{\mathrm{e}}$ siècle: les novices étaient appelés samaniy, du pāli sāmanera (Than Tun 1978, 94-95; voir aussi A dictionary of Buddhist terms 1996, 172-173). 
parfois attribuée: elle veille sur eux, les habille et les maquille, leur porte la nourriture à la bouche et leur essuie les lèvres. Plus fastueuse est la cérémonie, plus important est le montant des offrandes reçues, plus grands sont les honneurs rendus aux enfants, et plus grand sera également le prestige qui rejaillit sur l'assemblée. Le contraste avec le vœu de renoncement que font les enfants en fin de cérémonie n'en sera que plus accentué. Lorsque vient l'heure pour les jeunes princes de se faire raser le crâne et de porter la robe, la cérémonie laïque est pour eux terminée. Les bonzes et les membres de la famille se succèdent pour la séance de rasage. Lors de cette phase ultime du rituel, la plupart des invités s'en sont retournés et ne restent plus donc que les proches parents, les bonzes et les novices. L'hommage qui leur est alors rendu, par tous, jeunes ou vieux, parents et amis, change de nature. De princes auprès desquels on vient chercher grâce et protection, ils sont devenus membres de la communauté bouddhique dont on attend sagesse et enseignement. L'initiation bouddhique à proprement parler commence.

Je ne reviendrai pas ici dans le détail des cérémonies de prise de robe. Pour la seule Birmanie elles ont fait l'objet de nombreuses études (Shway Yoe 1963; Nash 1965; Spiro 1971; Aggasāra, 1995) et j'ai moi-même discuté longuement des formes rituelles et des enjeux sociaux contenus dans l'initiation bouddhique des adolescents (Robinne 2000). Mais, que ce soit dans l'État shan du Sud (Nyaung-Shwé) ou en Birmanie centrale (Pagan et Mandalay) où les enquêtes ont été effectuées, la cérémonie bouddhique de prise de robe comporte dans son déroulement une dimension laïque. C'est ce que j'appelle l'intermède laïque de l'initiation bouddhique au mariage.

\section{Variantes et constantes de l'initiation bouddhique}

Les variantes peuvent être très nombreuses d'une cérémonie de prise de robe à l'autre. Elles sont moins conditionnées par la distinction milieu urbain - milieu rural que par leur distribution en Birmanie centrale, à majorité birmane, ou dans l'État shan du Sud où l'influence taïe se fait plus fortement sentir.

\section{Sens et enjeux}

L'initiation reproduit, on le sait (Nash 1965; Spiro 1971), la scène de renoncement du bodhisattva. Comme dans toute cérémonie bouddhique, un important réseau d'entraide est activé à cette occasion. Des sommes importantes sont engagées à hauteur des enjeux sociaux que cela génère. Je ne reviendrai pas sur les implications sociologiques contenues dans la prise de robe, à savoir que le capital rituel des offrandes se conjugue au capital social du réseau d'entraide; mais en aucun cas la cérémonie de prise de robe ne saurait être associée à des fins lucratives comme semble l'admettre Nash $(1965,305)$. 


\section{Le lieu}

À Pagan et à Mandalay, un pavillon provisoire richement décoré (mandap, romanisé dans ce qui suit en manda) était construit au milieu d'une rue tandis que les shinbyu observés à Inlé se déroulaient dans la maison. Dans tous les cas, une prise de robe commence au monastère (ph. 1) avec la présentation aux bonzes des vêtements monastiques roulés dans le bol à aumône (Aggasāra, 1995, 4-5):

Vénérable bonze, afin de nous permettre de nous évader du cycle de la souffrance [samsāra dukkha], et afin de m'engager dans la Voie [magga] qui conduit au nirvana [nibbana] aussi vite que possible, accepteriezvous de me retourner cette robe et de m'initier?

C'est également au monastère que s'achève une cérémonie de prise de robe avec la cérémonie de rasage des cheveux et le prêche du bonze.

\section{La durée}

En milieu urbain, les cérémonies de prise de robe se déroulent le plus souvent sur une seule journée, trois si l'on compte le jour de construction du pavillon provisoire et l'offrande de nourriture au monastère suivie d'un prêche par le bonze. Le faire-part du village de Myinkaba à Pagan mentionne les trois jours: les $7^{\mathrm{e}}, 8^{\mathrm{e}}$ et $9^{\mathrm{e}}$ jour de la lune descendante du mois de Natdaw de l'année birmane 1356 (24-26 décembre 1994). Le faire-part du village de Kye-Za-Kun à Inlé mentionne également trois jours bien que le rituel s'échelonne en réalité sur sept jours, du $6^{\mathrm{e}}$ au $13^{\mathrm{e}}$ jour de la lune montante du mois de Natdaw de l'année birmane 1357 (décembre 1995). Des journées consacrées à la préparation matérielle du rituel (cuisson du riz et des ragoûts, etc.) avec distribution rigoureuse des tâches entre différents quartiers ou villages sont en effet intercalées entre le rituel aux esprits et le rituel bouddhique d'une part et, d'autre part, entre les différentes phases du rituel bouddhique.

\section{Le nombre des adolescents}

Le nombre d'enfants concernés peut également varier d'un shinbyu à l'autre. Le coût très élevé du rituel explique que les montagnards pao n'ont pas les moyens d'effectuer chaque année une cérémonie de prise de robe; à un niveau plus global de construction identitaire dans laquelle la Pao National Organisation et son corollaire la Pao Nation Army sont très activement engagées, les grands rassemblements et les réseaux engendrés contribuent pour beaucoup à cet écart de plusieurs années entre deux shinbyu: les cérémonies initiatiques des Pao peuvent ainsi réunir jusqu'à plusieurs centaines de novices originaires de différents villages. Sur le lac Inlé comme en Birmanie centrale, plusieurs rituels initiatiques sont en revanche organisés chaque année dans des villages voisins, voire dans un même village; le nombre d'enfants est proportionnellement plus réduit: sept à la cérémonie intha de Kye-Za-Kun, dix-sept à la cérémonie birmane du village de Myinkaba à Pagan. 


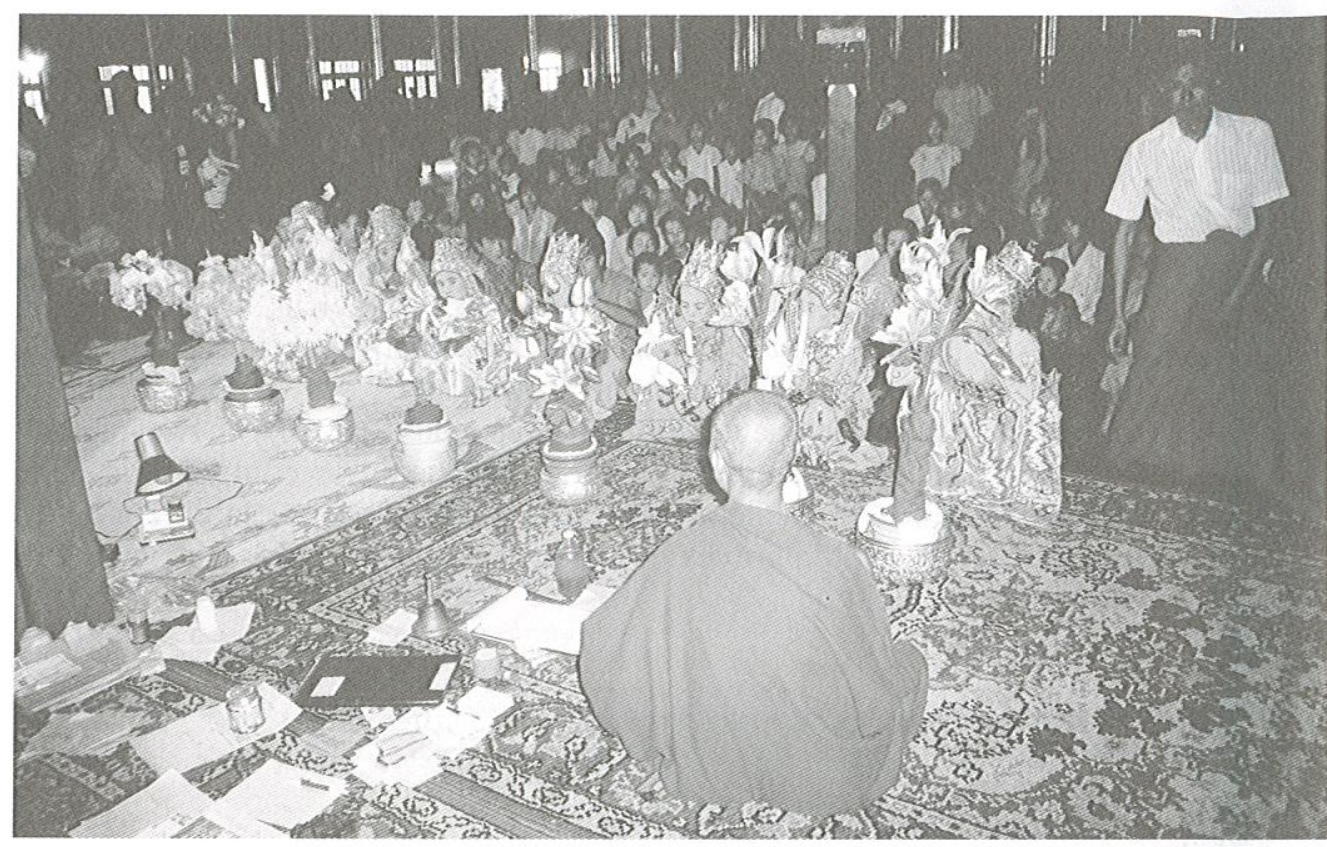

1 - Les jeunes princes présentent aux bonzes des vêtements monastiques roulés dans le bol à aumône (Inlé) (photo F. Robinne)

\section{La cérémonie de percement des oreilles}

La cérémonie de prise de robe des jeunes garçons s'accompagne le plus souvent d'une cérémonie de percement - réel à Inlé (ph. 7 p. 25) ou symbolique à Pagan et à Mandalay - des oreilles pour les jeunes filles. Cette cérémonie est appelée nâ:sa manigalā pwè, littéralement "la cérémonie auspicieuse d'embellissement des oreilles". Le nombre des jeunes filles - nā:sa, romanisé en nata - est souvent plus réduit que celui des novices: deux seulement à Kye-Za-Kun et quatre à Myinkaba. Nous préciserons plus loin le sens de cette cérémonie.

\section{La structure des cérémonies processionnelles}

Une prise de robe s'accompagne nécessairement de cérémonies processionnelles au cours desquelles les adolescent(e)s sont présentés au Bouddha, aux bonzes (ph. 2 p. 16), aux esprits, aux anciens. Une circumambulation fut ainsi effectuée autour des stūpas Shwezigon à Pagan et Mahāmuni à Mandalay, qui comptent parmi les plus prestigieux du pays. L'absence de stūpa aux proches environs du village de Kye-Za-Kun à Inlé explique que les trois déambulations soient effectuées autour du monastère. Dans tous les cas, celles-ci sont réalisées en gardant à main droite l'axe sacré. Les enfants sont vêtus en prince et portés dans les bras ou montés sur un moyen de transport. À Pagan comme à Mandalay, les garçons prirent place sur un cheval; les jeunes filles suivaient dans un cas à pied, dans l'autre sur des 
chars à bœufs de course richement décorés. Dans tous les cas, les enfants sont précédés des membres des familles hôtes, entourés devant et derrière de pucelles et de puceaux, ces derniers poussant le chariot musical. À Inlé, les shinlaung étant conduits en bateau (ph. 3), les arrêts sont plus difficiles à réaliser, ce qui explique que la présentation aux anciens soit effectuée de façon individuelle de maison à maison. Une dimension historico-culturelle est parfois donnée lorsque, comme à Mandalay, des troupes théâtrales représentent la cour birmane à différentes époques (Pagan, Ava, Amarapura), lesquelles sont alors indiquées par un panneau; le contraste de cette cérémonie processionnelle au milieu du trafic intense de la ville n'en est que plus saisissant. Malgré ces variantes, le principe des trois déambulations rituelles reste identique.

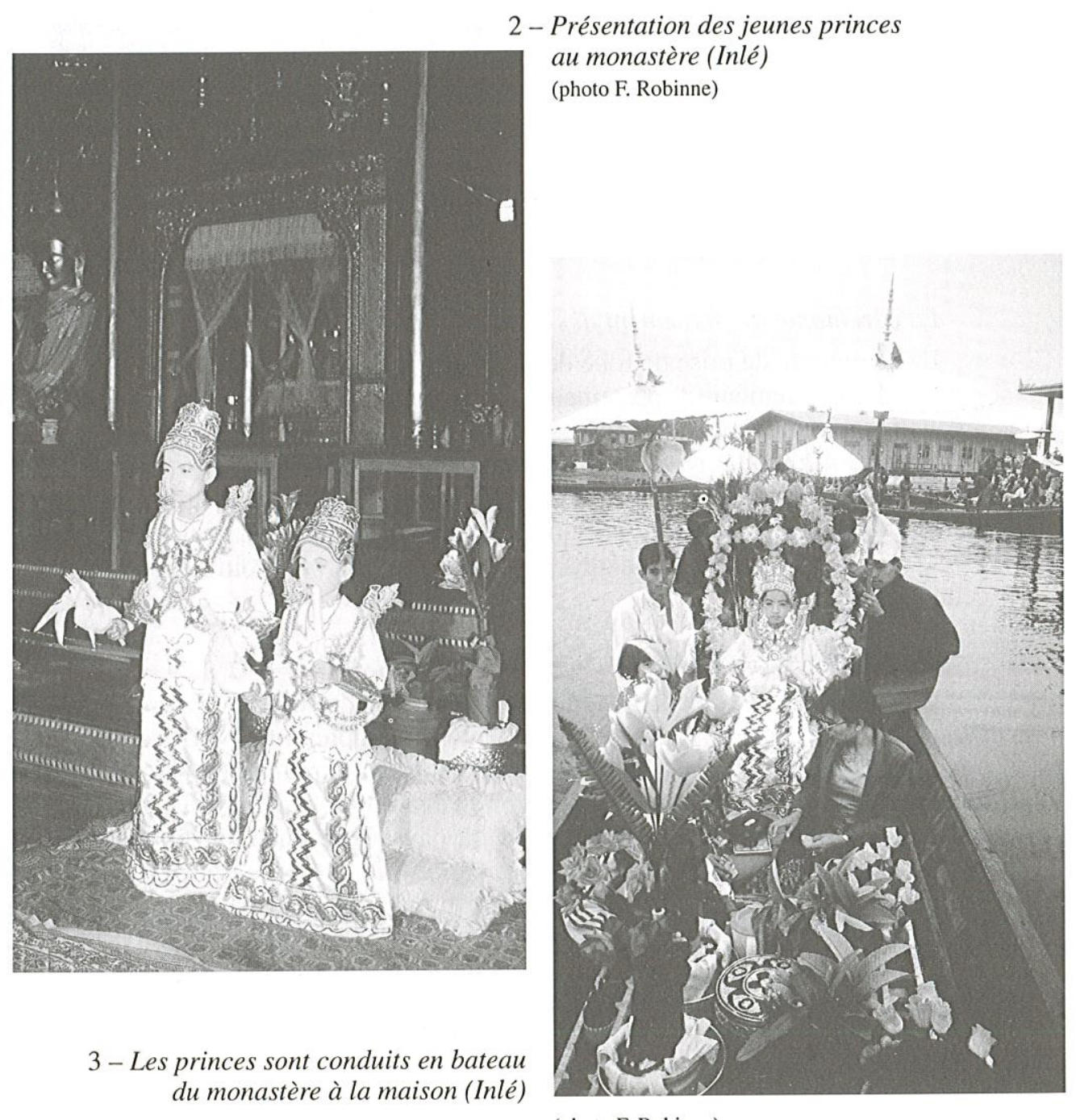

(photo F. Robinne) 
Tableau comparatif des rituels de Birmanie centrale et de l'État shan du Sud

\begin{tabular}{|c|c|c|c|}
\hline & $\begin{array}{c}\text { Birmanie centrale: } \\
\text { Myinkaba (Pagan) et Mandalay }\end{array}$ & $\begin{array}{l}\text { État shan du Sud: } \\
\text { Kye-Za-Kun (Inlé) }\end{array}$ & \\
\hline & & $\begin{array}{l}\text { Offrande aux nat: } \\
\text { Division spirituelle tripartite de } \\
\text { l'espace domestique: } \\
\text { - Autel du Bouddha; } \\
\text { - Autel des nat de l'intérieur; } \\
\text { - Autel des nat } \text { de l'extérieur. }\end{array}$ & $1^{\mathrm{er}}$ jour \\
\hline & & \multicolumn{2}{|c|}{$2^{\mathrm{e}}$ et $3^{\mathrm{e}}$ jours } \\
\hline $1^{\mathrm{er}}$ jour & $\begin{array}{l}\text { - Construction du manda dans la rue; } \\
\text { - cuisson du riz et des ragoûts; } \\
\text { - } \text { 1 }^{\text {re }} \text { déambulation autour du stūpa } \\
\text { (Shwezigon à Pagan, Mahamuni } \\
\text { à Mandalay); } \\
\text { - présentation à l'autel des esprits } \\
\text { (colline de Mandalay). }\end{array}$ & $\begin{array}{l}\text { Jour de l'appel des shinlaung: } \\
-6 \text { heures: présentation aux bonzes; } \\
\text { - } 8 \text { heures: présentation aux esprits } \\
\text { (offrande sur les îles délimitant } \\
\text { le monastère des villages); } \\
\text { - } \text { 1 }^{\text {re }} \text { déambulation du monastère } \\
\text { à la maison; } \\
\text { - les } 3 \text { rituels du seuil; } \\
\text { - présentation aux anciens. }\end{array}$ & $4^{e}$ jour \\
\hline $2^{\mathrm{e}}$ jour & $\begin{array}{l}\text { - Offrande à l'orchestre; } \\
\text { - } 9 \text { heures: déambulation autour } \\
\text { du quartier (Mandalay); } \\
\text { - } 11 \text { heures: offrandes de nourriture } \\
\text { aux bonzes dans le manda; } \\
\text { - } 14 \text { heures: séance d'habillage; } \\
\text { - présentation aux esprits tutélaires } \\
\text { (Pagan); } \\
\text { - 16 h } 30: 2^{\mathrm{e}} \text { déambulation autour } \\
\text { du village. }\end{array}$ & $\begin{array}{l}\text { Jour des cordons de coton: } \\
\text { - les offrandes aux shinlaung (avec } \\
\text { cordons de coton), } \\
\text { puis boucles d'oreilles aux nata; } \\
\text { - division sexuelle } \\
\text { de l'espace domestique; } \\
\text { - } 2^{\mathrm{e}} \text { déambulation } \\
\text { de la maison au monastère; } \\
\text { - séance de rasage l'après-midi et } \\
\text { prise de robe, puis } 1^{\text {re }} \text { initiation. }\end{array}$ & $5^{\mathrm{e}}$ jour \\
\hline $3^{\mathrm{e}}$ jour & $\begin{array}{l}\text { - } 9 \text { h } 30: 3^{\mathrm{e}} \text { déambulation autour } \\
\text { du village; } \\
\text { - séance d'initiation par le maître } \\
\text { de consécration; } \\
\text { - séance de rasage l'après-midi et } \\
\text { prise de robe, puis 1re initiation; } \\
\text { - prise à témoin de la Terre, en pré- } \\
\text { sence des bonzes sous le manda. }\end{array}$ & $\begin{array}{l}\text { Jour intermédiaire: } \\
\text { - cuisson du riz et des ragoûts; } \\
-3^{\mathrm{e}} \text { déambulation: transport des } \\
\text { offrandes de la maison au } \\
\text { monastère. }\end{array}$ & $6^{e}$ jour \\
\hline $4^{\mathrm{e}}$ jour & & $\begin{array}{l}\text { Le jour du prêche: } \\
\text { - offrandes de nourriture aux bonzes; } \\
\text { - prêche, puis prise à témoin } \\
\text { de la Terre au monastère; } \\
\text { - danse alternée des vierges. }\end{array}$ & $7^{e}$ jour \\
\hline
\end{tabular}

Les formes rituelles de Myinkaba à Pagan et de Mandalay étaient sensiblement similaires, notamment dans la durée et, on le verra, avec l'intervention d'un maître de consécration. Le tableau ci-dessus rappelle dans leurs grandes lignes les constantes et variantes entre les rituels de Birmanie centrale, d'une part, de l'État shan du Sud d'autre part. 
Dans tous les cas, la prise de robe fait l'objet d'une double initiation en fonction de ses deux composantes - la composante laïque lorsque les jeunes enfants sont faits princes (shinlaung), et la composante religieuse lorsque les princes font leur vœu de renonciation pour devenir novices (koyin). Cela est particulièrement clair lorsqu'il est fait appel à un maître de consécration, lequel officie dans le premier temps (à la maison ou sous un pavillon provisoire) tandis que le rituel est ensuite pris en charge par les bonzes: l'initiation monastique débute dans le cadre laïque de la maison avant d'être parachevée dans le cadre religieux.

Il est remarquable de constater que la présence d'un maître de consécration est commune aux deux rituels de prise de robe et de mariage et à eux seuls.

\section{L'intervention du maître de consécration}

À Pagan, à Mandalay, ou encore au village de Yeigyi dans la même région de Birmanie centrale (Spiro 1971, 241), il est fait appel à un spécialiste aussi bien pour la prise de robe que pour le mariage. Le statut de maître de consécration est à la portée de tous, à condition d'être un donateur émérite et de posséder quelque don pour la prédication, reconnaissance que l'âge suffit en général à conférer. Sa présence est indissociable de celle d'une troupe orchestrale, qu'il dirige ou avec laquelle il a pour habitude d'officier. À Pagan, les membres de l'orchestre étaient originaires de Kyauk Padaung, une ville située à quelques miles de Pagan. La mélodie jouée est appelée "le son de l'eau pure" (re cań sam), expression birmane qui désigne aussi l'eau offerte aux esprits (Myanmar-English Dictionary, 394). Les musiciens s'accordent pour dire qu'elle reproduit le son que produit l'eau d'un fleuve qui, de sa source, poursuit son cours jusqu'à l'embouchure. Cette métaphore de l'eau qui s'écoule peut être appliquée aux phases de la vie individuelle que sont dans leur continuité la naissance, la prise de robe et le mariage.

De préférence à l'expression "maître de cérémonie" utilisée par Nash $(1965,129)$ et par Spiro $(1971,241)$, j'emploie "maître de consécration" par référence au sens premier du mot transcrit en birman (bisik charā) et dérivé sous cette forme du pāli abhiseka (Myanmar-English Dictionary 1998, 321). À ma connaissance, il n'est pas fait appel à un tel officiant dans l'État shan du Sud. À quelques variantes près, précisées dans ce qui suit, les interventions du maître de consécration concentrent en un jour les phases rituelles réparties ailleurs sur plusieurs jours. Elles se succèdent comme suit:

1. Après quelques paroles de bienvenue (manigala $c a k \bar{a}$ ), le maître de consécration regarde sa montre - il a choisi lui-même l'heure, à la minute près, emprunte des signes les plus favorables dans l'acception astrologique du terme - et sonne alors de la conque (kharu). U Khyi Lhain, un des maîtres de consécration les plus renommés de Mandalay, explique qu'il y a deux sortes de conque: 
l'une dont la spirale tourne vers la gauche - celle utilisée pour le shinbyu - et l'autre dont la spirale tourne vers la droite utilisée de préférence - il n'en donne pas les raisons - pour un mariage. Le coquillage est généralement associé à Sakka, la divinité supérieure du panthéon bouddhique, pourvoyeuse des eaux de pluie lors du nouvel an birman, ainsi que dans les jātaka (Myint Swe 1994, 15 et 20). De l'eau est versée dans la conque. Qualifiée de pure, fraîche et indivisible, cette eau lustrale consacre l'union du novice à la communauté bouddhique comme elle consacrera plus tard l'union de deux époux (ph. 8 p. 28).

2. Invitation lancée au Bouddha et aux bonzes (bhurā: pañ. samighā) afin qu'ils honorent la cérémonie de leur éminente présence.

3. Rappel des cinq préceptes du bouddhisme (ngā: pā sīla) recommandant de respecter le Bouddha, le Dhamma, le Sangha, les maîtres et les parents puis, dans le cas de la prise de robe, des cinq engagements complémentaires (chay pä sïla): ne pas prendre de nourriture de midi jusqu'à l'aube le lendemain matin, ne pas assister à des spectacles et ne pas jouer d'instrument de musique, ne pas user de fleurs, de parfums ou de cosmétique, ne pas faire usage de grands sièges ou de grands lits, ne pas avoir d'affaires d'argent ${ }^{4}$.

4. Appel aux nat supérieurs - ou dewa - du panthéon bouddhique (parmi lesquels les gardiens des Quatre Directions) et à Wathundaye, la divinité de la Terre à l'intention de laquelle est consacrée la libation qui clôt toute cérémonie bouddhique, initiatique ou non. Le maître de consécration présente à trois reprises les plateaux d'offrandes, puis il se prosterne à trois reprises, enjoignant les shinlaung et les nata dans un cas, les jeunes mariés dans l'autre, à faire de même.

5. Appel aux nat inférieurs, aux Trente-Sept de l'extérieur, aux nat gardiens du village et aux nat individuels de ne pas perturber le bon déroulement de la cérémonie bouddhique.

6. Discours sur les offrandes à rendre aux nat (ahorapariboga en translittération, āhāraparibhoga en pāli). Ce thème est décomposé en trois phases:

- tenant dans la même main un bol de soupe de riz - "la nourriture des nat" précise le maître de consécration - et les cordons de coton enfilés entre ses doigts, il porte à trois reprises la nourriture à la bouche de chacun des shinlaung et des nata-comme l'ont fait à Inlé les "servantes" et comme le feront mutuellement les conjoints en renouvelant ce geste lors d'une cérémonie de mariage - puis le

4. Les dix conduites morales du novice, samaṇera sīla en pāli. 
maître de consécration leur souhaite une longue vie de plus de cent ans;

- il les invite à vénérer la religion en toutes circonstances;

- il leur rappelle que toute offrande de nourriture aux nat (natsudā) est un acte méritoire (ph. 4).

7. Comme en d'autres circonstances rituelles, initiatiques ou non, des fils de coton protecteurs sont présents aux deux cérémonies de prise de robe et de mariage. Lors d'une prise de robe, l'officiant - bonze ou maître de consécration - réunit sept ou onze fils de coton en un seul toron. Il récite ensuite des versets ${ }^{5}$ censés protéger des onze sortes de souffrance, puis des formules (gāthā) ponctuées d'un "Om". Ces paroles et le pouvoir qu'elles contiennent sont supposés passer au travers des cordons réunis en un seul filin, tout comme à Inlé les cordons étaient passés d'un seul tenant autour du cou des novices tandis que les bonzes auxquels ils étaient ainsi symboliquement reliés récitaient les versets bouddhiques. L'officiant enroule alors autour du cou de chacun des shinlaung ce collier de coton (ph. 5), de même qu'à Inlé (ph. 6) les bonzes effectuent successivement ces deux étapes (collective puis individuelle) des fils de coton passés au cou des novices ${ }^{6}$. Leur fonction est double: transmettre les paroles sacrées prononcées par l'officiant (médium, bonze ou maître de consécration) et éloigner les forces du mal, d'où leur nom de parit kruī:, "fils de protection". Des fils écrus peuvent de la même façon être passés au cou des conjoints, non pas cette fois au monastère, mais dans la maison où se tient la cérémonie de mariage. Il peut s'agir aussi d'une bobine non déroulée. Elle est déposée dans un plateau à offrandes, face aux bonzes puis face au maître de consécration. Ce n'est qu'après la cérémonie qu'une longueur de fil pourra être emportée par chacun afin d'en entourer l'enceinte de sa maison.

La présence des fils de coton n'est spécifique ni à la prise de robe ni au mariage. Ils peuvent accompagner toute cérémonie auspicieuse: j'ai personnellement observé cela lors de la naissance d'un enfant à Pagan, lors du nouvel an birman à Mandalay, ou encore lors d'une fête de monastère près de Thibaw, dans l'État shan du Nord, au cours de laquelle le Patthana était récité. Néanmoins le dédoublement de la séance au niveau individuel puis collectif, au monastère dans un cas, à la maison dans l'autre, marque autant un passage qu'il scelle dans les deux cas une union.

5. Parit khya, de parita en pāli.

6. La phase rituelle des cordons de coton passés autour de la communauté bouddhique est également pratiquée au Laos (Condominas 1998) et en Thaïlande (Terwiel 1994, 40, 194-195 et 230-232; Ku Daeng 1976, 214 et fig. 22). 


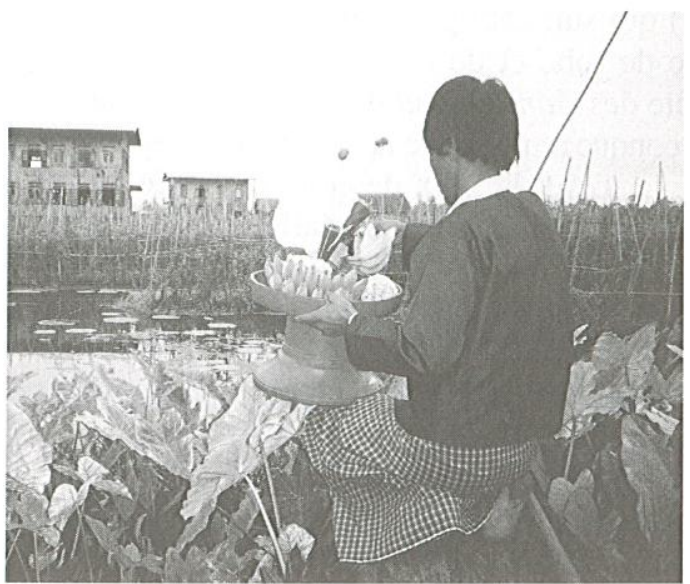

4 - Offrandes aux nat en périphérie $d u$ monastère (Inlé)

(photo F. Robinne)

5 - Fils de coton passés au cou des jeunes princes lors d'un shinbyu (Pagan) (photo F. Robinne)

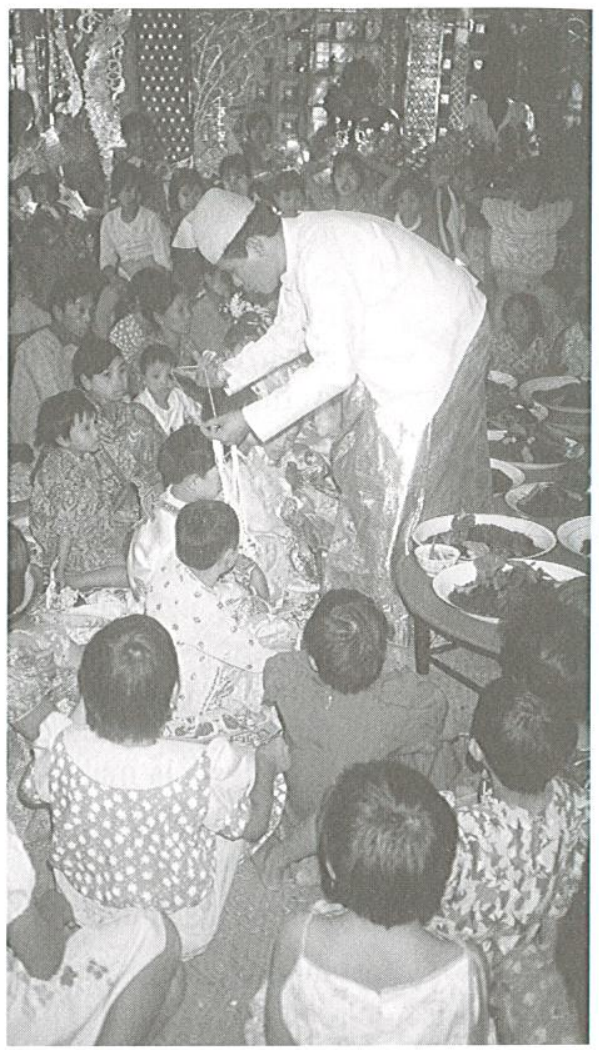
(photo F. Robinne)

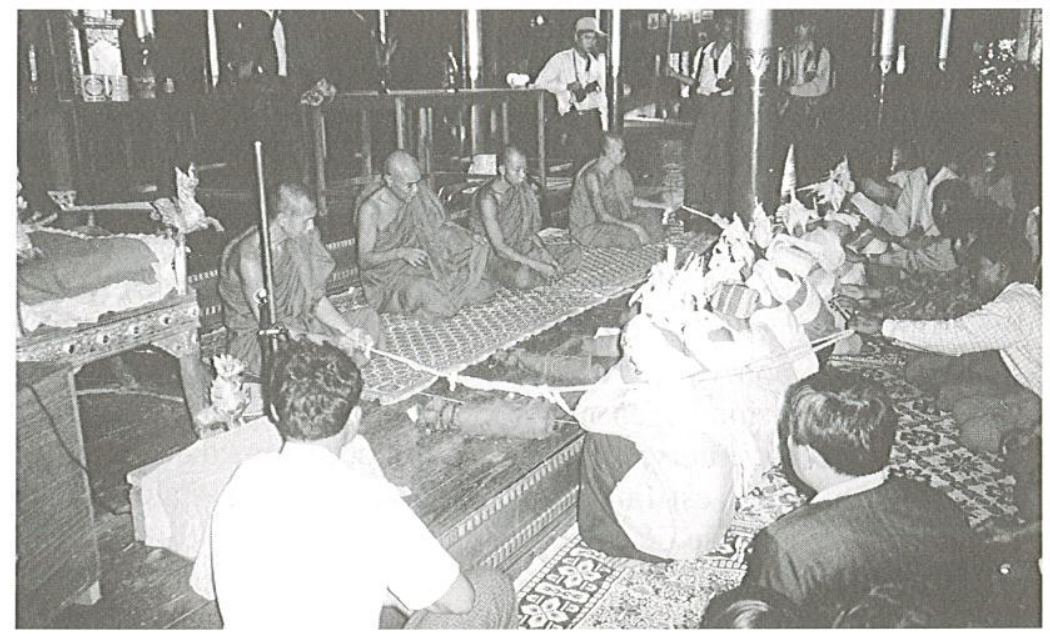


8. L'ablution rituelle (recan swan:) qui suit est également commune aux deux cérémonies de prise de robe et de mariage. Le maître de consécration asperge la tête des shinlaung et des nata à l'aide de feuilles d'Eugenia dans la conque remplie d'eau dont il ne se sépare pas. Le restant d'eau est versé sur la noix de coco disposée au centre des trois mains de bananes, opération que réalise également le vieux maître de cérémonie d'Inlé, en utilisant cette fois des feuilles de Crocus trempées dans l'eau dont il asperge tout d'abord les deux bols symbolisant la Mère gardienne du Nord, puis les couvre-chefs cérémoniels des shinlaung et de la jeune nata. À Pagan et à Mandalay, un assistant du maître de consécration s'empare alors d'un gâteau de riz (de couleur rougeâtre à cause de la mélasse qu'il contient et surmonté de riz éclaté de couleur blanche) et va l'offrir aux nat gardiens du village. À Inlé, le maître de cérémonie s'est également occupé de la préparation des deux plateaux d'offrandes aux nat de la maison, offrandes présentées à trois reprises et dans lesquelles se retrouvent également le rouge et le blanc: la farce introduite à l'intérieur des deux poissons frits se compose d'un mélange de riz blanc et de lentilles, et les deux sortes de galettes sont l'une rouge et l'autre blanche, comme les deux sortes de gâteaux de riz. Ces deux couleurs symbolisent respectivement les esprits de l'intérieur - par référence au panthéon bouddhique - et les esprits de l'extérieur associés à la male mort (Robinne 2000, 189-192).

9. Les shinlaung comme les conjoints s'emparent d'un bol à aumônes - laqué noir lors d'un shinbyu, en argent lors d'un mariage - afin de quêter dans l'assistance.

C'est au monastère que s'achève le rituel initiatique, avec la séance de prise de robe à proprement parler. Les shinlaung ont d'abord le crâne rasé par les bonzes, les parents récupérant les cheveux des enfants, reproduisant en cela le geste de Sakka lorsqu'il recueillit les cheveux du Bouddha pour les enfouir dans le stūpa Sulamani. Mais tandis que les cheveux du Bouddha sont de précieuses reliques sur lesquelles sont construites les stūpas, ceux des futurs novices seront jetés quelques instants plus tard dans un endroit quelconque. L'officiant, bonze et/ou maître de consécration, rappelle les règles de conduite que doivent observer les novices comme les devoirs réciproques des deux conjoints seront par la suite énumérés.

De même que toute cérémonie débute ou s'achève par une offrande de nourriture aux bonzes en l'absence du maître de consécration, la présence de ce dernier n'est indispensable ni à l'initiation bouddhique ni au mariage. C'est ainsi qu'il n'intervient jamais en ces occasions dans la région d'Inlé. Toutefois, lorsqu'il est fait appel à lui, sa présence - alors réservée aux seuls mariage et prise de robe - fait bien plus que souligner la similitude 
des deux rituels. Elle contribue tout à la fois à renforcer la part de laïcité dans la cérémonie de prise de robe et la part de religiosité dans le mariage. Son intervention contribue de fait à accentuer l'alternance entre la dimension laïque et la dimension religieuse contenues dans chacun des deux rituels.

\section{Intermèdes laïques de l'initiation au mariage}

\section{Échanges amoureux alternés}

Chacune des interventions du maître de consécration est marquée par une mise en scène musicale. Les membres de l'orchestre entament une parodie de la vie quotidienne: parodie des jeunes épris l'un de l'autre, mais également parodie des bonzes dont ils imitent le mode de récitation des textes bouddhiques. L'effet comique auprès de l'assistance est assuré. Bien que la séance ne dure que quelques heures, on y retrouve le mode opératoire en tout point similaire aux intermèdes laïques qui se succèdent sept jours durant à Inlé.

Lors des cérémonies de prise de robe observées à Pagan et à Mandalay, l'intervention du maître de consécration était non seulement très structurée, mais les différentes phases rituelles étaient séparées l'une de l'autre par un intermède laïque prenant la forme d'un échange alterné (achak asway) entre le maître de consécration et l'orchestre. Chacun de ces intermèdes est un spectacle en soi, au cours duquel se succèdent chansons et dialogues comiques sur le thème des jeux amoureux. La fin de l'intermède est saluée par les shinlaung et les nata qui se tournent vers l'assemblée devant laquelle ils se prosternent à trois reprises, les mains jointes devant le visage.

Le thème des relations amoureuses est constitutif de tous les shinbyu, tout comme la division sexuelle et la séparation dans l'union constituent des composantes structurelles de ces rituels. Bien qu'exprimées différemment en Birmanie centrale et dans le Sud de l'État shan, les dimensions bouddhiques et laïques de la prise de robe sont une constante, de même que l'échange alterné sur le thème des relations amoureuses. Car si le shinbyu est avant tout un rituel bouddhique, c'est autant une initiation à l'enseignement du Bouddha qu'une initiation au mariage.

Cette dimension est renforcée dans le cas intha en deux occasions qui sont deux phases complémentaires du rituel. Lors de la troisième et dernière déambulation rituelle au cours de laquelle les offrandes sont conduites de la maison au monastère, les jeunes garçons interpellent les filles de la région sur le thème "Nous avons beaucoup de belles-sœurs, mais il reste encore au moins autant de filles à marier". Le lendemain soir, après que le prêche du bonze a clos le rituel initiatique à proprement parler (c'est-à-dire dans sa dimension bouddhique), la fête ne fait que commencer pour les membres des quatre familles ayant contribué au shinbyu. C'est dans la maison des 
principaux donateurs que les jeunes garçons et les jeunes filles, amis proches et ou membres des familles organisatrices du shinbyu, se retrouvent le soir pour une compétition de danse. Par conséquent, tandis que les novices passaient leur première nuit au monastère, ceux-là entamaient la danse des vierges. Les garçons entreprennent tout d'abord en solo ou par deux une série de danses, faisant à chaque fois prouesse d'imagination et d'agilité pour attirer l'approbation des filles. Elles leur répondent de la même manière. Un couple garçon-fille danse parfois ensemble. Les filles refusent rarement l'invitation à danser, mais elles tancent parfois le prétendant d'un: "Marie-toi donc dans l'année avec une veuve!", donnant ainsi la réplique, par un jeu d'alternance différée, aux chants impétueux des garçons la veille (Robinne 2000).

L'idée d'échanges amoureux entre les garçons et les filles lors d'une cérémonie de prise de robe n'est pas propre aux Intha. Hla Pe (1985, 180181) rapporte ainsi à propos des Birmans des fredaines que la prise de robe inspirait aux jeunes filles mélancoliques. Voici un des chants que la petite amie chante à son amoureux la veille de la cérémonie du noviciat:

As I walk to the East

And gaze West over my shoulder,

I see the pagoda with its gold pennons and streamers

Holding in your arms a bamboo wrapper full of writings

You are getting ready to enter the monastery

What are you going to study

How many lents will you be spending

Please tell your beloved in your own sweet voice

Lors d'un shinbyu, la formule utilisée au moment de porter la robe est identique à celle employée au moment de passer les fils de coton lors d'une naissance; kap tay, littéralement "coller". Dans l'acception rituelle correspondante, il est ainsi fait référence, dans un cas, à l'appartenance nouvelle à la communauté bouddhique dans son rapport à la communauté laïque que l'on vient de quitter, et, dans l'autre cas, à l'autonomie désormais effective de l'âme du nourrisson par rapport à celle de sa mère. Selon un processus similaire à celui réalisé lors du "collage" de l'âme papillon d'un nouveau né ou du "collage" de la robe d'un novice, processus qui consiste à distinguer pour mieux identifier, la séparation hommes/femmes affichée tout au long de ce rituel est sous-jacente au rapprochement des sexes.

\section{La prise de robe des garçons et le percement des oreilles des filles}

Les enfants, garçons et filles, sont assis sur le "trône". Les invités se succèdent pour leur faire des offrandes d'argent et passer des cordons de coton autour du cou de chacun d'eux. La dernière à se présenter devant les shinlaung et la nata est l'infirmière du village de Min-Khyaung Est. En charge des soins courants, elle officie aussi en tant que sage-femme, et 


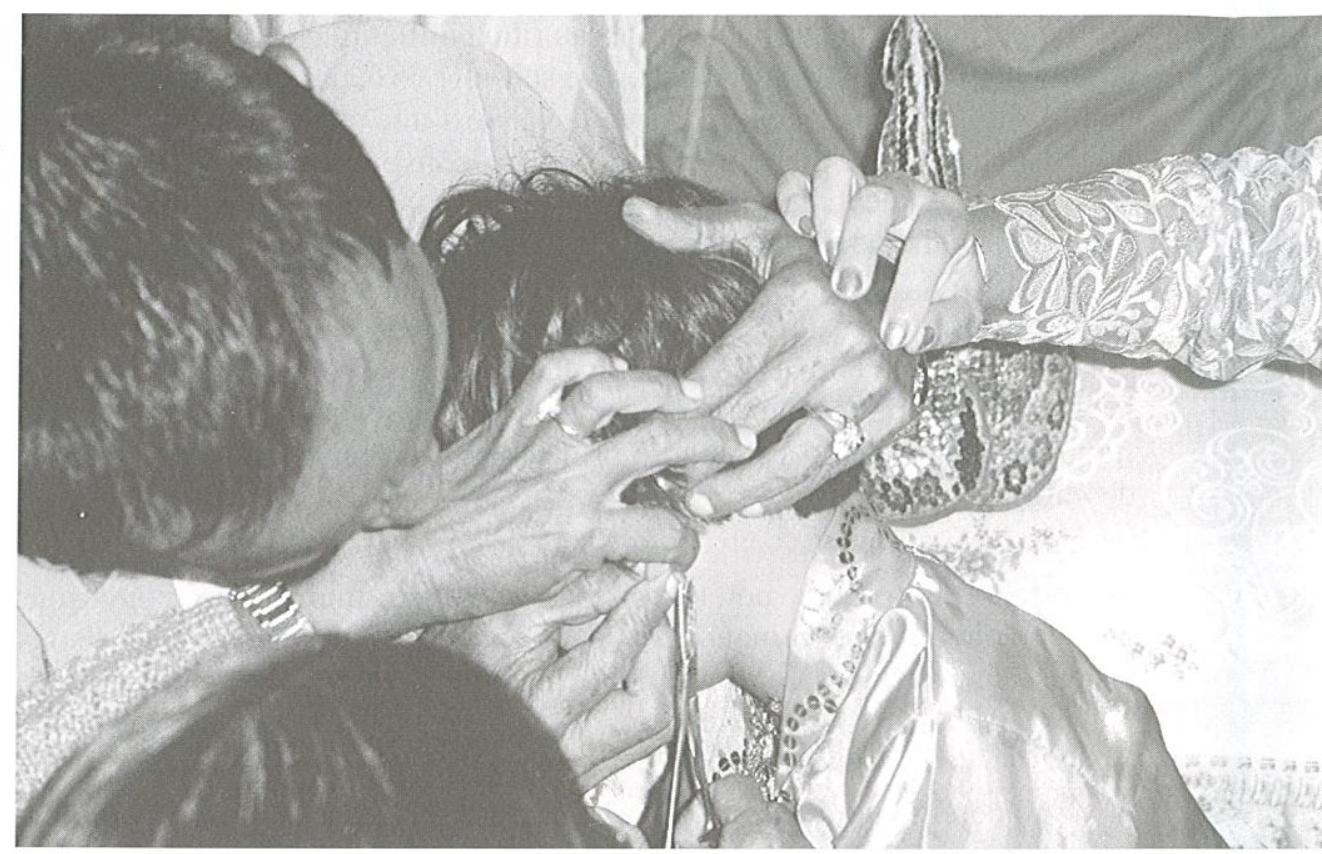

7 - Percement des oreilles d'une jeune fille

lors d'un shinbyu (Inlé) (photo F. Robinne)

c'est à elle qu'est confié le percement des oreilles. Âgée d'à peine trois ans, la trop jeune nièce n'a pu participer à la cérémonie d'offrandes, comme l'a fait son aînée. Le percement des oreilles est expédié aussi rapidement que le permettent les hurlements des deux fillettes qui se débattent.

Dans un style qui témoigne de sa proximité avec le quotidien birman, Shway Yoe $(1963,48-49)$ fait remarquer à juste titre que:

The first great event in a Burmese girl's life is the nā-twin mingala, the boring of the ears. [...] That ceremony is much an epoch to her as putting on the yellow robe, or getting his legs tattoed, is to her brother. Ever afterwards she will look upon every male as a possible lover. No more taking alternate whiffs at a big green cheroot with little Maung Po; no more teasing Po Shan to let her have a taste of those fine neetè mangoes his father has got down from Mandalay; no more dawdlings about the streets by herself. [...] In a word, the nā-twin mingala transforms the girl into a woman, just as much as admission to a monastery makes the boy a man.

À Pagan comme à Mandalay, le maître de consécration effectue symboliquement le rasage du crâne des adolescents en soufflant sur leur tête. Et c'est tout aussi symboliquement qu'il perce l'oreille des jeunes filles en touchant du doigt le lobe de chaque oreille. 
La participation des jeunes filles au rituel initiatique des jeunes garçons commence et s'achève ici. Aussi brève soit-elle, la cérémonie du percement des oreilles est une étape importante de la vie d'une jeune fille. Parée de ces boucles et pourvue grâce à elles d'oreilles convenablement arrangées, la jeune nata est devenue dans l'absolu une jeune femme potentiellement mariable. Le thème se retrouve dans les chroniques birmanes (Pe Maung Tin et Luce 1960,84-85) comme dans les mythes fondateurs shan, pao et taungyo de la région de Nyaung-Shwe (Robinne 2000 et à paraître). Identifiées comme étant des reliques du Bouddha remises par le roi birman Anawratha, les boucles d'oreilles élèvent les jeunes femmes - généralement issues de minorités ethniques - au rang de princesses. Un préliminaire obligé pour devenir épouses de roi.

Lorsque les jeunes filles viennent d'être élevées au rang d'épouse potentielle, leur époux potentiel s'engage l'après-midi de ce même jour dans la voie du renoncement. Les deux conditions à un mariage placé sous les meilleurs auspices, l'une rédhibitoire et l'autre essentielle, sont remplies.

\section{Division sexuelle de l'espace}

Une cérémonie d'offrandes aux nat est effectuée trois jours avant que ne commencent les trois jours du rituel initiatique à proprement parler. Bien qu'elle fasse partie du cours normal du rituel de prise de robe, cette cérémonie d'offrandes aux nat n'est pas annoncée dans le faire-part. Elle ne réunit que les intimes, ainsi que le maître de cérémonie, un villageois de Kye-Za-Kun Sud, vieil oncle par alliance du maître de maison, réputé pour ses connaissances en la matière.

Plusieurs autels des nat sont répartis au deuxième niveau de la maison, celui où se trouve la pièce consacrée à l'autel du Bouddha ainsi que, séparées par une cloison, les parties privées. L'autel de la Mère gardienne du Nord est pour l'occasion déplacé du pilier Sud-Est au pilier Nord-Est. Ce déplacement annonce une bipolarisation de l'espace plus générale qui se maintiendra pendant tout le déroulement du rituel: les autels des nat de l'extérieur sont éloignés de ceux des nat de l'intérieur - les seuls nat à qui soit alors appliqué le qualificatif de "gardiens de la Religion" - et des photographies de bonzes qui entourent l'autel du Bouddha; l'espace dévolu au faste qui entoure les jeunes princes est ainsi démarqué de l'espace dévolu au bouddhisme et au renoncement, l'objet même du rituel initiatique. Le même principe de séparation conduira à réserver l'espace privé aux esprits de l'extérieur, aux princes et finalement aux femmes, l'espace collectif étant quant à lui réservé aux images du Bouddha, aux futurs novices et aux hommes.

Ces féminisation et masculinisation des deux pièces sont une création aussi ponctuelle qu'artificielle, nécessitée, une fois de plus, par le dédoublement du rituel réalisé ce jour-là: cérémonie de percement des oreilles le matin et prise de robe l'après-midi. 


\section{La reconnaissance sociale du mariage}

\section{La cérémonie d'apposition des mains}

Considérée dans la dimension matrimoniale qui lui est sous-jacente dans chacune de ses phases rituelles, la cérémonie d'initiation transforme symboliquement l'état de virginité des enfants des deux sexes: une fois initiés, ils deviennent socialement des conjoints potentiels. Après la reconnaissance de l'aptitude spirituelle au mariage acquise lors d'une prise de robe ou du percement des oreilles qui l'accompagne généralement - au moins symboliquement, on l'a vu -, la cérémonie de mariage proprement dite est avant tout sanctionnée par une reconnaissance sociale de la parentèle et du voisinage.

Trois expressions birmanes peuvent être employées pour signifier "se marier". La plus commune est im thon pru tay dont le sens littéral est "dresser la maison". Sur les faire-part de mariage, l'expression utilisée est marigalā chon tay, littéralement "construire, réaliser la chance", car un mariage ou un shinbyu sont nécessairement auspicieux, le sens de mañgalā.

La troisième expression couramment utilisée pour signifier "se marier" est lak thap tay (mot à mot "main + apposer + marque modale"). L'apposition des mains des deux conjoints au cours de la cérémonie du mariage est en effet le symbole par excellence du mariage. Les deux mains droites sont mises à plat l'une sur l'autre et sont enserrées par une étoffe de coton symbolisant l'union(ph. 8 p. 28). Le maître de consécration verse de l'eau sur les mains ainsi réunies, quand il ne les trempe pas dans le bol à aumône argenté, signifiant ainsi que "leur union sera aussi indivisible que l'eau" (Spiro 1986, 184). Après avoir précisé que les bonzes seraient extrêmement scandalisés s'il leur était demandé de participer à ce rituel, voici ce que dit Shway Yoe à propos de cette séquence rituelle propre au mariage (1963, 57):

The old custom that the bride and bridegroom should join their right hands together, palm to palm, in the presence of all the assembled guests, and then should eat rice out of the same dish and feed each other with one or two morsels in turtle-dove fashion, has in many cases died out, and the eating and drinking, the talk of the men in the main room of the house and the women in the inner apartment, is quite enough to solemnise the union.

Toutefois, un autre rite symbolise tout aussi fortement l'union, il s'agit du franchissement des seuils commun au shinbyu et au mariage.

\section{Le franchissement des seuils}

Au cours d'un mariage, comme à l'occasion d'une prise de robe, des rituels dits "du seuil" sont effectués. Ils consistent dans les deux cas à franchir un barrage de chaînes d'or tendues au travers de la porte de la maison par de jeunes pucelles. Deux ou trois seuils doivent être successivement franchis par les adolescents ou les jeunes mariés (ph. 9 et 10 p. 29): 
8 - Maître de consécration

officiant lors d'un mariage (Mandalay) (photo F. Robinne)

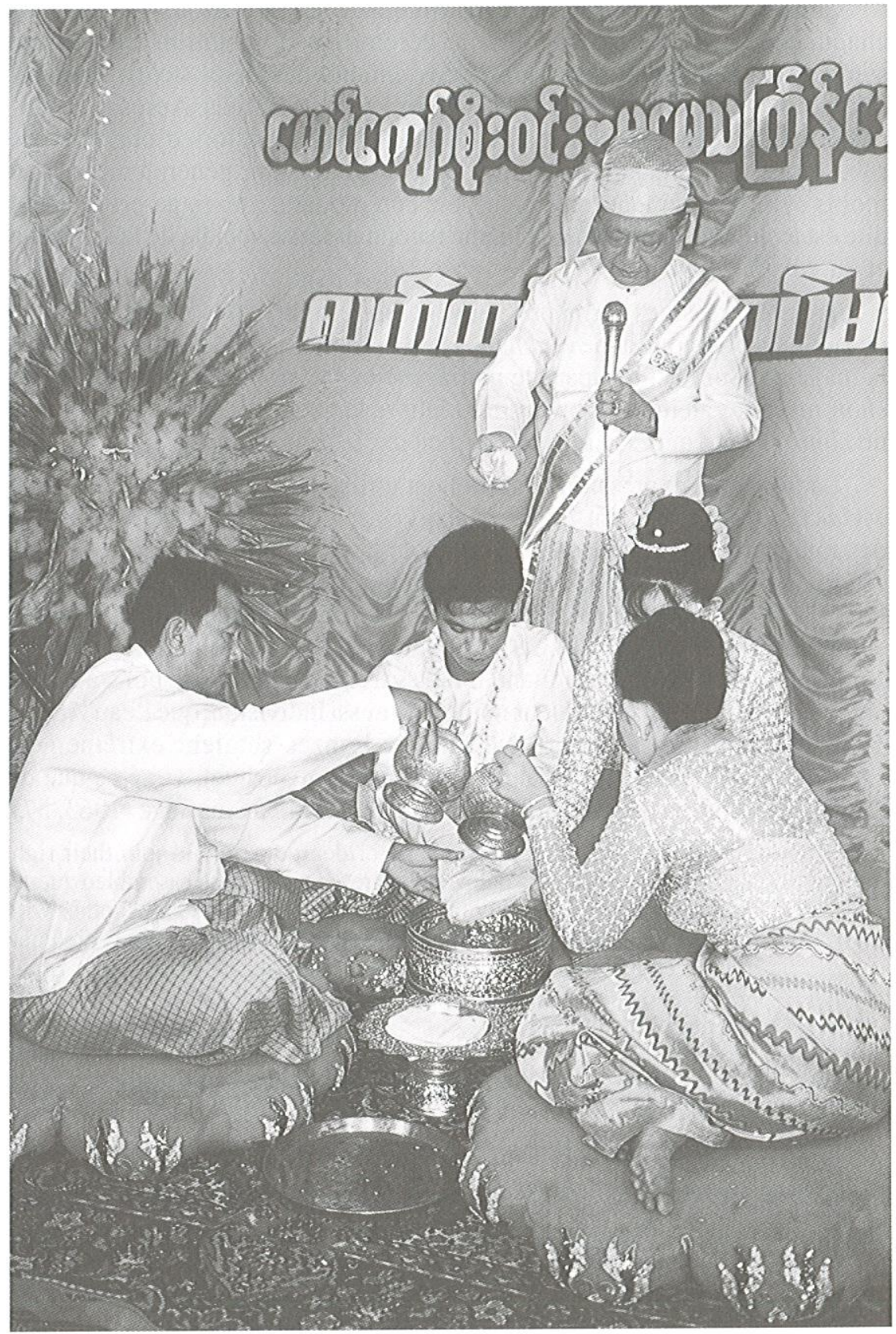


9 - Franchissement du seuil lors d'un mariage (Mandalay) (photo F. Robinne)

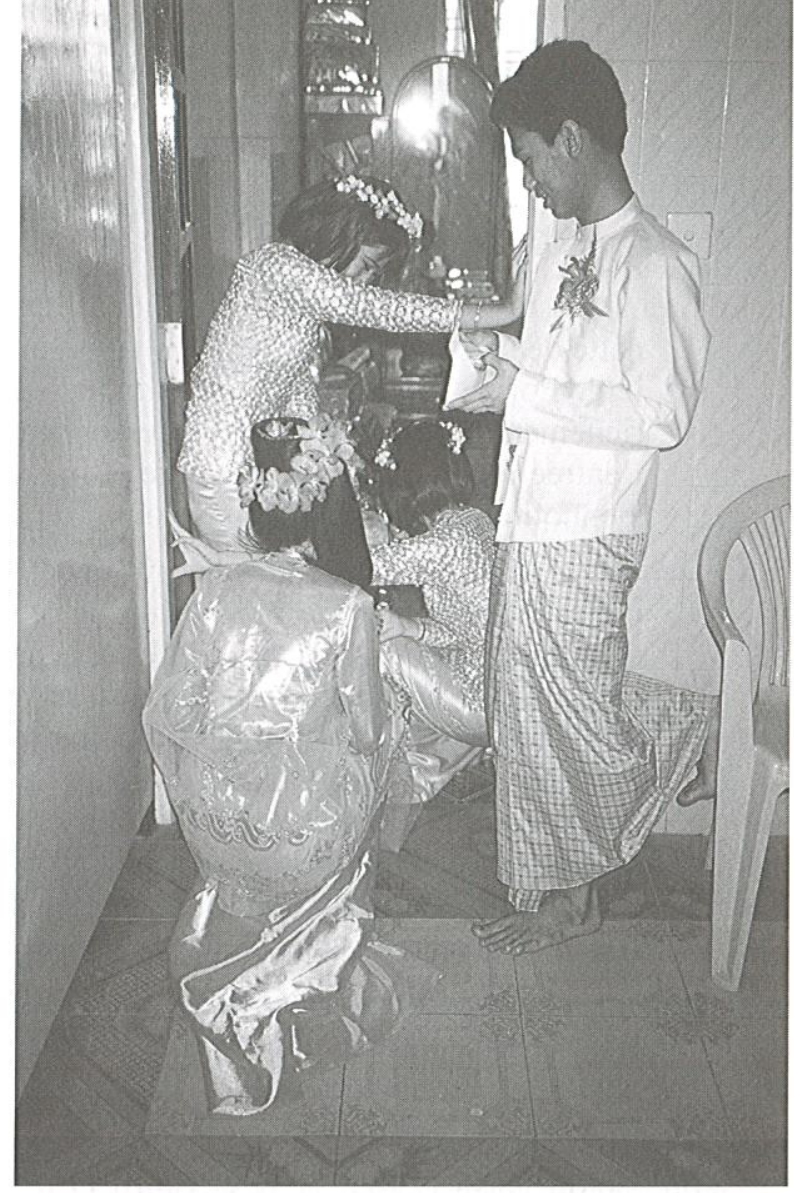

\section{0 - Franchissement} du seuil lors d'une prise de robe (Inlé) (photo F. Robinne)

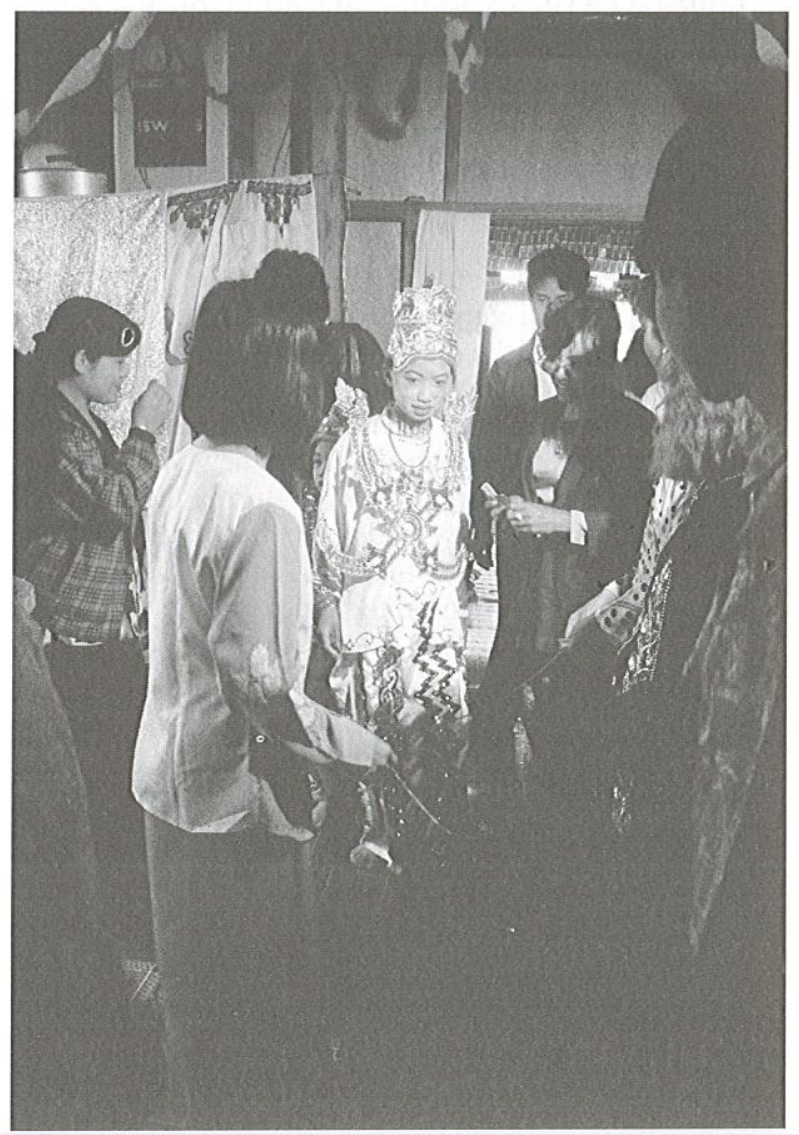


1. Le premier est un arrêt très bref effectué, à l'extérieur, sur une simple natte de bambou recouverte d'un tapis sur lequel du riz éclaté est jeté. Forme simplifiée d'un manda - le pavillon provisoire richement décoré que certains dressent en pareille circonstance à l'entrée de leur maison - la natte et le tapis ont la même valeur symbolique de quatre points autour d'un centre;

2. Le second arrêt, moment de franche rigolade, est identique au rituel pratiqué lors d'un mariage avant que le couple n'entre dans la chambre nuptiale. Ayant pris place sur le pas de porte de la maison, des femmes, nécessairement vierges, se succèdent par deux dans un ordre d'âge décroissant au fur et à mesure que l'on monte les marches. Elles ferment l'accès de l'escalier à l'aide d'une chaînette en or tendue au travers de l'escalier ou en se donnant la main. Le passage n'est libéré que lorsque les "servantes" distribuent au nom des shinlaung une somme d'argent jugée suffisante. À chaque fois la surenchère déclenche des éclats de rire;

3. Il leur faut ensuite franchir un second barrage de colliers dorés, tendus au travers de la porte par huit jeunes femmes.

Ces franchissements du seuil de la maison ou du pavillon provisoire sont sanctionnés immanquablement par une offrande d'argent, une "rançon" selon Nash $(1965,251)$ et Spiro $(1986,185)$. La dimension ludique de ce rite explique sans doute qu'il s'observe aussi bien en milieu urbain qu'en milieu rural, dans les hautes terres comme dans les basses terres. Le terme birman est khè bhuī:, littéralement "le prix de la pierre". Shway Yoe $(1963,57-58)$ associe ce "prix de la pierre" à une pratique ancienne et sans doute tombée en désuétude, que, pour ma part, je n'ai jamais eu l'occasion d'observer:

The other custom is much more singular, more ancient and infinitely more disagreable. On the night of the marriage a band of young bachelors of the place come and shower stones and sticks on the roof of the house where the happy couple are, keeping up sometimes such a sustained battery that the thatch or wooden shingles suffer materially, while the furniture and even the newly-wedded couple occasionally do not escape injury. In Lower Burma the lads are usually bought off with a sum of money, and where the officials do not interfere to prevent it, the custom has degenerated into a system of extortion. [...] The money paid is supposed to be devoted by the receivers to making offerings at the pagoda [...]. In Upper Burma the blackmail is never demanded.

Différentes explications en sont données. Nash (1965, 251-252) corrobore pour la haute Birmanie (région de Mandalay) cette pratique de chaîne tendue en travers d'un seuil, ainsi que sa sanction éventuelle par un jet de pierre si les intéressés ne paient pas une somme d'argent. Cet auteur analyse à juste titre que: 
It may be, in part, a survival of that custom, for the payment is indeed higher if the groom comes from another village, but mainly it is a contribution to the peer group as compensation for their work as ushers, servers, cooks, and cleaners at the wedding and is a source of the funds that maintain the unmarried as an organized segment of village social structure.

Spiro $(1965,185-186)$ - qui se réfère vraisemblablement à Forbes cité par Shway Yoe $(1963,58-59)$ - associe ce prix de la pierre à un mythe bouddhique que lui racontèrent les villageois de Yeigyi:

According to the myth, this custom originated after the recurrent destructions of the earth and all its inhabitants by fire, water and wind. Sometimes after this destruction, four gods (samma deva), beings without sex organs, descended to the earth where, finding no food, they discovered that soil was a source of nourishment. Having ingested this terrestrial matter, they became more material and were thus unable to ascend to their heavenly abode. Soon after, they discovered a certain wild-growing root, as well as wild rice, which they added to their diet. As a result, they began to develop sex organs, as well as those "mental impurities" (kilesa) which Buddhism identifies as greed, anger, and lust; and this in turn led them to the practice of sexual intercourse. When the higher gods (brahma deva) observed them engaging sex, they became angry and stoned them.

Du point de vue développé ici, il est intéressant de constater que cette pratique commune à la prise de robe et au mariage revêt une connotation sexuelle, quel que soit le caractère prédominant de la cérémonie - païen ou bouddhique.

Les bonzes auprès de qui j'ai enquêté à Mandalay s'accordent pour dire que, pour être reconnu, le mariage n'a pas besoin de cérémonie religieuse, pas même d'une offrande de nourriture aux bonzes. Seul l'accord des deux parties est indispensable. Il existe encore de nombreux mariages par "enlèvement" - khui yu tay en birman (littéralement "appeler et enlever"). De manière générale, le couple ayant ainsi agi obtient rapidement gain de cause, que la vie commune soit sanctionnée ou non par une cérémonie.

\section{L'offrande aux bonzes}

Une cérémonie de mariage est généralement associée à la seule cérémonie laïque effectuée au domicile de l'un des parents ou dans une salle de réception louée pour l'occasion. Les invités se succèdent dans la matinée autour des tables où sont servis quelques gâteaux et boissons. Le jeune couple passe de table en table saluer les convives et se faire photographier auprès d'eux. Le faste donné à la cérémonie n'est pas proportionnel à la reconnaissance sociale. Dans bien des cas, la vie conjugale sans autre artifice suffit à acquérir l'aval de son entourage. Il n'en reste pas moins que la dimension symbolique de l'union s'efface derrière la dimension festive du mariage. 
C'est la raison pour laquelle les auteurs s'accordent globalement pour dire que le mariage n'est pas lié au bouddhisme. "The ritual is very simple and has nothing whatever of a religious character about it", avance Shway Yoe $(1963,57)$. Tandis que Maung Maung déclare $(1963,54)$ : "Marriage is a civil institution into which the Buddhist religious element enters not at all". Dans le même sens, Manning Nash $(1965,251)$ écrit pour sa part: "The interdiction on marriages during the period of Wa [carême bouddhique] is strictly observed. The Buddhist monk is never present, not even as spectator; none of the devotions in the village liturgy are chanted; no reference is made to the Buddha and his dhamma". Plusieurs éléments semblent corroborer ces dires, dont certains textes bouddhiques. L'histoire - attribuée au Bouddha dans le verset 202 du Dhammapada - des deux jeunes époux est de ce point de vue éloquente (Mya Tin 1995, 338):

While residing at the Jetavan monastery, the Buddah uttered Verse (202) of his book at the house of a lay-disciple, with reference to a young bride. On the day a young woman was to be wedded to a young man, the parents of the bride invited the Buddha and eighty of his disciples for alms-food. Seeing the girl as she moved about the house, helping with the offering of alms-food, the bridegroom was very much excited, and he could hardly attend to the needs of the Buddha and the other bhikkhus. The Buddha knew exactly how the young bridegroom was feeling and also that time was ripe for both the bride and the bridegroom to attain Sotāpatti Fruition. By his supernormal power, the Buddha willed that the bride would not be visible to the bridegroom. When the young man could no longer see the young woman, he could pay full attention to the Buddha, and his love and respect for the Buddha grew stronger in him. Then the Buddha said to the young man, "O young man, there is no fire like the fire of passion; there is no evil like anger and hatred; there is no ill like the burden of the five aggregates of existence (khandhas); there is no bliss like the Perfect Peace of Nibbāna." $[\ldots]$ At the end of the discourse both the bride and the bridegroom attained Sotāpatti Fruition.

Néanmoins, l'affirmation selon laquelle le mariage n'est pas corrélé à une cérémonie bouddhique mérite pour le moins d'être nuancée. Sur ce chapitre, je rejoins l'analyse de Spiro $(1986,183)$ :

Although the wedding, like the engagement, is an entirely secular ceremony - Buddhist monks do not officiate, nor may they attend even as guests - it is framed by religion, a Buddhist ceremony being performed in the morning prior to the wedding, and a spirit (nat) ceremony in the evening (or the following morning) after the wedding.

Le mariage a pourtant fait l'objet d'un traitement très pointu dans certains textes bouddhiques. Le Dictionnaire de la nature humaine rédigé en birman dresse une liste des cérémonies de bon augure censées ponctuer le parcours individuel. Un total de douze est mentionné. Il s'agit du premier jour de fécondation, du jour de naissance, des $3^{\mathrm{e}}$ et $7^{\mathrm{e}}$ jour après la naissance, de la 
cérémonie du berceau, du $75^{\mathrm{e}}$ jour après la naissance, de la cérémonie du premier bétel passés 75 jours, du $3^{\mathrm{e}}$ mois, du $100^{\mathrm{e}}$ jour correspondant à la dation du nom, du $6^{\mathrm{e}}$ mois associé au premier riz, de la cérémonie du chignon, du percement des oreilles, de la prise de robe et enfin du mariage (Obādā Bhi Wam Sa s.d., 539-540).

Par ailleurs, les Dhammathat ou Lois bouddhiques, au nombre de trente-six en Birmanie, accordent de nombreux développements sur les différentes sortes de pucelles - jusqu'à vingt et une kañña, apyui en birman (U Gaung 1909, 25) -, sur les différentes sortes mariages āvāha ou vivāha selon que la fiancée est conduite au fiancé ou inversement - mais le nombre va jusqu'à huit (U Gaung 1909, 35), sur la classification des femmes en douze, vingt et une ou vingt-deux classes (U Gaung 1909, 30) ou réduite comme les hommes en cinq catégories (U Gaung 1909, 137) ou en fonction de leur rang dans la hiérarchie, les différentes sortes d'épouses et d'époux et leurs cinq devoirs respectifs (U Gaung 1909, 208-209). Les lois bouddhiques sont très détaillées concernant le mariage et tout ce qui peut en découler: adoption, divorce, héritage, etc. Selon la même source, les textes de loi bouddhiques Mano, Kaingza, Kandaw, Vannadhamma ou Rāsì précisent que l'âge des mariés doit être de quinze ou seize ans, c'està-dire peu de temps après la cérémonie de prise de robe ${ }^{7}$. Selon le Temiya jātaka cité par le texte de loi Mānussika, "Personne ayant atteint l'âge de la puberté n'est soustrait au désir naturel d'aimer et d'être aimé, de posséder ce qui est désirable" (U Gaung 1909, 32-33).

En tant qu'acte méritoire par excellence, la cérémonie bouddhique est une valeur ajoutée, facultative, à la cérémonie laïque. Lorsqu'il est fait appel aux bonzes, ceux-ci n'interviennent jamais durant la cérémonie. Cela transparaît dans les faire-part de mariage. À l'occasion du mariage de Ta-Min-Thein-Naing et de Ma-Khin-Shwé-Sin le 29 novembre 2000 à Mandalay, le faire-part se composait d'une première invitation à participer à l'offrande très auspicieuse de nourriture aux bonzes (mañgalä chwam: kap pwè phit krā: $l h w \bar{a})$ - la proche famille et les amis étaient invités à se rendre le $13^{\mathrm{e}}$ jour de la lune descendante du mois de Tan-Zaung-Mon de l'ère birmane 1362, de huit heures à dix heures du matin, dans la maison des parents de la jeune femme -; la seconde invitation (thim: mrā: mangalā añ. khamं pwè phit krā: $(h w \bar{a})$ correspondait à la cérémonie laïque, laquelle se tient tantôt dans une salle louée pour l'occasion, tantôt sous un manda construit provisoirement, comme pour une prise de robe, sur la rue en face de la maison.

7. Mendelson (1975, 165-167) a par exemple montré que, sur les 171 novices de l'enquête, $60 \%$ furent ordonnés entre les âges de dix et quinze ans.

8. La graphie des auteurs cités est respectée. 
Lors d'un autre mariage, la cérémonie débuta par une offrande de nourriture aux bonzes effectuée tôt le matin, à six heures - "so that the karma of the couple [should] rise like the rising sun" selon Spiro $(1986,183)-$, dans la maison des parents de l'un des deux conjoints. Le supérieur entama un prêche au cours duquel il témoigna de l'hommage rendu aux bonzes (kandō), rappelant ensuite les cinq règles que doit suivre tout laïque et la rétribution karmique qui rejaillit sur tout un chacun. La cérémonie s'acheva avec la prise à témoin de la Terre. Un repas fut ensuite offert aux bonzes, avant que ceuxci ne s'en retournent. La cérémonie laïque orchestrée par le maître de consécration débuta quelque deux heures plus tard sur cette harangue:

Aujourd'hui, le $10^{\mathrm{e}}$ jour de la lune montante du mois de Tazaungmon, en 1362 de l'ère birmane, le dimanche 5 novembre $2000 \mathrm{AD}$, jour placé sous les meilleurs signes, à $9 \mathrm{~h} 12$, heure de bon augure, débute la cérémonie de mariage de Maung Kyaw Swar Min et de Ma Ni Kay Khaing.

Bien que les deux phases de la cérémonie se soient toutes deux déroulées au domicile et bien qu'un très court laps de temps ait séparé dans le second cas, l'offrande aux bonzes de la consécration laïque, les deux événements n'étaient pas dans l'absolu conçus dans la continuité l'un de l'autre. Comme pour le shinbyu un décalage est au contraire entretenu pour distinguer la cérémonie religieuse de la cérémonie laïque: la première dominant dans le premier cas tandis que la seconde prime dans le second cas.

En une occasion seulement, il m'a été donné d'assister à une cérémonie qui se tenait exclusivement dans un monastère de Mandalay. La raison en était que les parents de l'un des conjoints ne consentaient pas au mariage. De ce fait, la reconnaissance sociale fut limitée à quelques proches des deux côtés, mais la cérémonie était investie d'une forte dimension bouddhique. Cinq bonzes et cinq novices étaient présents. Les jeunes époux, qui se tenaient d'abord assis côte à côte face aux bonzes, vinrent se placer de part et d'autre de la table ronde basse où avaient été disposés les différents plats, puis ils soulevèrent légèrement la table en signe d'offrande respectueuse. Cela fait, ils récitèrent ensemble les versets protecteurs (andaray kañ parit). Après un moment de détente autour de plateaux à cigares, d'ustensile à bétel et de feuilles de thé mariné, les jeunes époux se rapprochèrent de nouveau des bonzes et des offrandes furent remises aux bonzes (outre une somme d'argent, des produits nécessaires à la vie quotidienne tels que tubes dentifrice et savon). La cérémonie de prise à témoin de la Terre fut alors effectuée. Après quoi la communauté laïque entama le repas offert par les hôtes.

Ce cas de figure d'un mariage dans le monastère est exceptionnel. La cérémonie d'offrande de nourriture aux bonzes - au domicile et/ou au monastère - participe du concept karmique, que l'acte méritoire soit ou non associé à une circonstance cérémonielle particulière. En milieu bouddhique, 
le mariage est effectivement une cérémonie secondaire, voire facultative. Les dimensions laïques et religieuses sont en général distinguées dans l'espace et dans le temps. Mais celles-ci ne sont pas pour autant exclusives l'une de l'autre: elles sont consubstantielles à la cérémonie de prise de robe comme à la cérémonie de mariage. Si la reconnaissance sociale de l'union prime sur le rituel bouddhique, c'est que l'aptitude spirituelle au mariage a été consacrée lors du rituel initiatique de prise de robe. Et si le déroulement de chacun des deux rituels montre que c'est dans l'alternance de la phase rituelle monastique et de la phase rituelle laïque que se déroulent la cérémonie de prise de robe et la cérémonie de mariage, c'est qu'elle renvoie elle-même aux formes alternées d'union et de séparation contenues dans les deux rites de passage.

\section{Repères bibliographiques}

A Dictionary of Buddhist Terms [Buddhadesanātō wohāra abhidham ]

2540 [1996] - Yangon, Ministry of Religious Affairs, 337 p.

(En anglais, birman et pāli)

Aggasāra, [vénérable]

1995 - [The Initiation of Novicehood], Rangoun, sacnuwecap, iii +117 p. (En birman et anglais)

CONDOMINAS, Georges

1980 - Le bouddhisme au village. Notes ethnographiques sur les pratiques religieuses dans la société rurale lao (plaine de Vientiane), Paris, EFEO, 230 p.

(En français et en lao)

Gaung, [U]

1909 - A Digest of the Burmese Buddhist Law concerning inheritance and marriage; being a collection of texts from thirty-six Dhammathats, compared and arranged under the supervision of $U$ Gaung, Rangoon, Goverment Printing, 2 vols.

GENNEP, Arnold van,

1981 - Les rites de passage. Études systématiques des rites, [1e éd. 1909] Paris, Éditions A. et J. Picard, 288 p. 
Hla Pe, [U]

s.d. - "A shin-byu-bwè in Burma", Literature, Historiography, Scholarship, Language, Life and Buddhism, London, Institute of Southeast Asian Studies, p. 178-185.

JUDSON, Adoniram

1966 - A Burmese-English Dictionary, Rangoon, Baptist Board [1st ed. 1852] of Publications, $1120 \mathrm{p}$.

KINGSHILL, Konrad

1976 - Ku Daeng-The red tomb. A village study in Northern Thailand AD 1954-1974, Bangkok, Suriyaban Publishers, $\mathrm{xx}+404 \mathrm{p}$.

Maung Maung, [U]

1963 - Law and Custom in Burma and the Burmese Family, The Hague, Martinus Nijhoff, xii + 155 p.

MENDELSON, E. Michael

1975 - Sangha and state in Burma: a study of monastic sectarism and leadership, Ithaca, New York, Cornell University Press.

Myanmar-English Dictionary

1998 - Yangon, Ministry of Education, Department of the Myanmar Language Commission, xxiii +365 p.

Mya Tin, [Daw]

1995 - The Dhammapada. Verses and Stories (translated by -),

[1st ed. 1985] Yangon, Myanmar Pitaka Association, xxix + 500 p.

Myint Swe, [U]

1994 - Jātkrī: chay bhwè [The ten great Jataka stories], Yangon, Mandalay, nawarat cāpe, 164 p. (texte birman) +174 p. (texte anglais).

NASH, Manning

1965 - The Golden Road to Modernity. Village Life in Contemporary Burma, New York, London, Sydney, John Wiley, viii + 333 p.

Obādā Bhi Wam Sa, [vénérable]

s.d. - Saruppra abhidham [Dictionnaire de la nature humaine], phyāpum tuik san, saraktō tuik, 820 p. (En birman) 
ROBINNE, François

2000 - Fils et maîtres du Lac. Relations interethniques dans l'État shan de Birmanie, Paris, CNRS/MSH, 364 p.

À paraître - "Early Burmese myths and history as revised by the minorities in their oral tradition".

Shway Yoe

1963 - The Burman. His Life and Notions, New York,

[1st ed. 1882] The Norton Library, xviii +609 p.

SPIRO, Melford E.

1971 - Buddhism and Society. A Great Tradition and its Burmese Vicissitudes, London, George Allen \& Unwin Ltd., xiv + 510 p.

1986 - Kinship and Marriage in Burma. A Cultural and

[1st ed. 1977] Psychodynamic Analysis, Berkeley, Los Angeles, London, University of California Press, $x i x+313$ p.

TERWIEL, B. J.

1994 - Monks and magic. An analysis of religious ceremonies

[third ed.] in Central Thaïland, Bangkok, White Lotus, xviii + 302 p.

Than Tun, [U]

1978 - History of Buddhism in Burma, AD 1000-1300, Rangoon, Journal of the Burma Research Society, Vol. LXI, Parts I \& II, v + 262 p.

François Robinne

CNRS

Maison Asie-Pacifique Institut de recherche sur le Sud-Est asiatique 
